\title{
Monitoring the service-based system lifecycle with SALMon*
}

\author{
Marc Oriol, Xavier Franch, Jordi Marco \\ Universitat Politècnica de Catalunya, Barcelona (Spain), GESSI research group. \\ \{moriol,franch\}@essi.upc.edu,jmarco@cs.upc.edu
}

\section{Resumen}

Los Sistemas Basados en Servicios (SBS) son sistemas software altamente dinámicos compuestos por un conjunto de servicios web provenientes de distintos, y posiblemente heterogéneos, proveedores. En contraste con otros sistemas software tradicionales, el comportamiento dinámico de los SBS requiere de información actualizada sobre la calidad de servicio (QoS) para poder actuar i administrar correctamente las actividades en las distintas fases del ciclo de vida de los SBS (p.e., selección de servicios, despliegue, evaluación de niveles de acuerdo de servicio -SLA-, y adaptación).

La necesidad de proveer esta información referente a la QoS ha resultado en distintas soluciones tecnológicas construidas alrededor de un componente software de monitorización (abreviadamente, monitor). Sin embargo, los monitores actualmente disponibles en el estado del arte han sido diseñados para apoyar sólo un subconjunto de las actividades del ciclo de vida del SBS.

Para cubrir esta brecha de investigación, presentamos SALMon, una plataforma de monitorización de servicios versátil que provee información acerca de la QoS según la forma y enfoque adecuado para las distintas actividades del ciclo de vida.

Para ello, hemos identificado los requisitos de las distintas actividades mediante (1) el análisis de las necesidades descritas en la literatura y (2) reuniones y entrevistas con miembros de distintos grupos de investigación que trabajan en dichas actividades.

A partir de estos requisitos, hemos desarrollado SALMon. Uno de los principales obstáculos es que los requisitos de monitorización para cada tarea son distintos, y en algunos casos, pueden ser contradictorios. Esto nos ha llevado a diseñar SALMon como un sistema modular, capaz de conectar los distintos módulos de manera que permita configurar la forma final del monitor según las necesidades de cada proyecto.

Los principales avances y características que SALMon provee son los siguientes:

- Combinación de estrategias de monitorización pasiva y testing online.

- Combinación de estrategias de configuración basado en transformación de modelos y mediante invocación directa.

- Extensibilidad mediante el añadido de nuevos atributos de calidad.

- Bajo acoplamiento con las tecnologías de los servicios monitorizados.

- Alta interoperabilidad.

(*) Artículo publicado en Expert Systems with Applications, 42(19), 2015. (I.F.: 2.24) 
Para validar nuestra propuesta, hemos integrado SALMon con plataformas que cubren las actividades del ciclo de vida anteriormente descritas. Para ello, hemos realizado un conjunto de colaboraciones con otras universidades y centros de investigación, donde SALMon ha sido integrado con sus herramientas. En particular:

- WeSSQoS [1] (Selección de servicios): Sistema experto capaz de tratar con distintas estrategias de selección para servicios web según la QoS requerida.

- FCM [2] (Despliegue): Sistema experto para la toma de decisiones en cuanto a la mejor estrategia de despliegue en un sistema de federación de clouds.

- SALMonADA [3] (Validación de SLAs): Una plataforma capaz de identificar y reportar violaciones de SLAs con la descripción de las causas de violación.

- MAESoS [4], PROSA [5], PROTEUS [6], CARE [7] (Adaptación): Plataformas que dan soporte a SBS adaptativos, capaces de definir y ejecutar adaptaciones en respuesta a un mal funcionamiento o fallo de los servicios del sistema.

\section{Agradecimientos}

Este trabajo ha sido parcialmente financiado por el gobierno español mediante los proyectos CICYT TIN2010-19130-C02-01 y TIN2013-44641-P; y por la Unión Europea mediante la red de excelencia de servicios S-Cube, número de contrato 215483.

\section{Referencias}

[1] O. Cabrera, M. Oriol, X. Franch, J. Marco, L. López, O. Fragoso, R. Santaolaya. Open framework for web service selection using multimodal and configurable techniques. In Computación y Sistemas (CyS). 2014

[2] A. Kertész, G. Kecskemeti, M. Oriol, P. Kotcauer, S. Acs, M. Rodríguez, O. Mercè, A. Cs. Marosi, J. Marco, X. Franch. Enhancing Federated Cloud Management with an Integrated Service Monitoring Approach. In JGC, 11(4), 2012.

[3] C. Müller, M. Oriol, X. Franch, J. Marco, M. Resinas, A. Ruiz-Cortés, M. Rodríguez. Comprehensive Explanation of SLA Violations at Runtime. In TSC, 7(2), 2013. [4] X. Franch, P. Gruenbacher, M. Oriol, B. Burgstaller, D.Dhungana, L. López, J. Marco, J. Pimentel. Goal-driven Adaptation of Service-Based Systems from Runtime Monitoring Data, in Procs. of COMPSACW, 2011.

[5] O. Sammodi, A. Metzger, X. Franch, M. Oriol, J. Marco, K. Pohl. Usage-based online testing for proactive adaptation of service-based applications, in Procs. of COMPSAC, 2011.

[6] E. Schmieders, A. Micsik, M. Oriol, K. Mahbub, R. Kazhamiakin. Combining SLA prediction and cross layer adaptation for preventing SLA violations, in Procs of. $2^{\text {nd }}$ WoSS, 2012.

[7] M. Oriol, N. Qureshi, X. Franch, A. Perini, J. Marco. Requirements monitoring for adaptive service-based applications, in Procs. of REFSQ, 2012. 